\title{
Optimization of Deposited Coating Chemical Composition during Operation in Urea Medium
}

\author{
Aleksandr Nazarko ${ }^{1, *}$ and Roman Plomodyalo ${ }^{1}$ \\ ${ }^{1}$ Kuban State Technological University, 2, Moskovskaya Str., the City of Krasnodar, 350072, Russia
}

\begin{abstract}
The article deals with the problem of optimization of the chemical composition of the deposited metal operating in the urea melt. To improve the technological properties and increase the resistance of the deposited metal against intergranular corrosion, manganese (0.5-1.8 pts. wt.) and niobium (0.3-1.8 pts. wt.) were introduced. The chromium content was varied in the range of 20-30 pts. wt., nickel $-3-8$ pts. wt.
\end{abstract}

\section{Introduction}

Austenitic-ferritic deposited coatings have a combination of properties such as good weldability, high resistance against intergranular corrosion and corrosion cracking, which makes them promising structural materials for surfacing the sealing surfaces of check valves, pump parts and other equipment operating in any kind of aggressive medium [1].

The choice of materials for surfacing equipment parts still remains an unsolved problem. When manufacturing equipment operating in acidic corrosive medium, it is recommended to use austenitic surfacing materials. However, when working in an alkaline medium, the deposited coatings made of austenitic wires as filler material, undergo selective corrosion along the fusion line [2]. When using filler materials identical to the chemical composition base metal, selective corrosion occurs in the deposited weld metal.

\section{Research Procedure}

Surfacing was carried out on plates of steel $03 \mathrm{Kh} 17 \mathrm{~N} 13 \mathrm{M} 3 \mathrm{~T}$ with the dimensions of 40x40x20 mm with experimental cast rods of the PNL-04Kh27N7M3D2B type [3] with a diameter of $5 \mathrm{~mm}$ and a length of $270 \mathrm{~mm}$, in argon medium, with 180 A of current

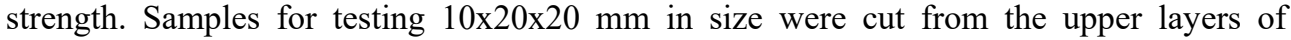
multilayer surfacing.

Heat treatment of the deposited samples was carried out in the PM-12M2P-1200 muffle furnace in the modes set in operation [4].

Corrosion resistance tests were carried out on samples (thermally treated [4]) under the conditions of the production process (urea melt) (Table 1).

\footnotetext{
* Corresponding author: nazarkoaleksandr@rambler.ru
} 
Table 1. Test Results

\begin{tabular}{|c|c|c|c|c|c|}
\hline Try No. & $\begin{array}{c}\mathbf{X}_{\mathbf{1}} \\
(\mathbf{C r})\end{array}$ & $\begin{array}{c}\mathbf{X}_{\mathbf{2}} \\
\mathbf{( N i )}\end{array}$ & $\mathbf{X}_{\mathbf{3}} \mathbf{( N b )}$ & $\mathbf{X}_{\mathbf{4}} \mathbf{( M n )}$ & $\mathbf{K}, \mathbf{g} /\left(\mathbf{m}^{\mathbf{2}} \cdot \mathbf{h}\right)$ \\
\hline 1 & 28.35 & 3.80 & 0.58 & 1.12 & $0.2284 ; 0.1996 ; 0.1919 ; 0.2162 ; 0.2706$ \\
\hline 2 & 21.70 & 7.70 & 1.35 & 1.70 & $0.3383 ; 0.4187 ; 0.4428 ; 0.3982 ; 0.3734$ \\
\hline 3 & 23.20 & 5.65 & 1.24 & 1.50 & $0.2798 ; 0.2827 ; 0.2640 ; 0.2645 ; 0.3057$ \\
\hline 4 & 19.76 & 7.40 & 1.50 & 1.32 & $1.2785 ; 1.3723 ; 1.2818 ; 1.3393 ; 1.2984$ \\
\hline 5 & 22.60 & 6.14 & 0.95 & 1.60 & $0.6041 ; 0.6092 ; 0.6570 ; 0.6587 ; 0.3461$ \\
\hline 6 & 27.45 & 4.30 & 0.55 & 1.70 & $0.3794 ; 0.3580 ; 0.3455 ; 0.3218 ; 0.3461$ \\
\hline 7 & 20.80 & 7.06 & 1.57 & 1.25 & $0.9761 ; 0.9269 ; 0.9709 ; 0.9679 ; 0.9344$ \\
\hline 8 & 28.20 & 5.28 & 1.10 & 0.72 & $0.2210 ; 0.2041 ; 0.2110 ; 0.2210 ; 0.2110$ \\
\hline 9 & 21.45 & 3.02 & 0.42 & 1.00 & $0.6694 ; 0.6157 ; 0.6541 ; 0.6571 ; 0.6981$ \\
\hline 10 & 25.40 & 3.50 & 0.45 & 0.80 & $0.3032 ; 0.3027 ; 0.2881 ; 0.3057 ; 0.3020$ \\
\hline 11 & 23.90 & 6.60 & 1.33 & 0.60 & $0.2857 ; 0.3206 ; 0.3548 ; 0.3349 ; 0.2928$ \\
\hline
\end{tabular}

\section{Research Results and Discussion}

The corrosion rate of the deposited metal is taken as an optimization parameter.

The calculations were performed in two stages. At the first stage, the dependence of the corrosion rate of the deposited metal in the urea melt on the content of alloying elements was determined. At the second stage, the chemical composition of the deposited metal was optimized.

To process the experimental results, the approximation method by analyzing pair correlation was applied [5]:

$$
K_{i}=f\left(x_{i}\right)
$$

Dependence of the corrosion rate of the deposited metal on the chromium content (Fig. 1 , a) is as follows:

$$
K_{1}=a_{1} x_{1}^{3}+b_{1} x_{1}^{2}+c_{1} x_{1}+d_{1}
$$

where $a_{1}, b_{1}, c_{1}, d_{1}$ - least square coefficients [6]. Dependences (Fig. 1, b-d) for nickel $\left(K_{2}\right)$, manganese $\left(K_{4}\right)$ and niobium $\left(K_{3}\right)$ were determined in a similar way. According to the theory of multiple correlation, the set of equations represents a mathematical model of the dependence of the corrosion rate of the deposited metal on the chemical composition:

$$
K=\sum_{i=1}^{4} K_{i}\left(X_{i}\right),
$$

where $K_{i}$ - corrosion rate of the deposited metal depending on the content of the $i^{\text {th }}$ element, $\mathrm{g} /\left(\mathrm{m}^{2} \cdot \mathrm{h}\right) ; i$ - number of optimization parameters.

Thus, the mathematical model has the following form:

$$
K_{0}=A_{0}+A_{1} X_{1}^{3}+B_{1} X_{1}^{2}+C_{1} X_{1}+B_{2} X_{2}^{2}+C_{2} X_{2}+B_{3} X_{3}^{2}+C_{3} X_{3}+B_{4} X_{4}^{2}+C_{4} X_{4} .
$$

Owing to the solution of the equation system on a personal computer and determination of the coefficients, the following dependence of the corrosion rate of the deposited metal on the chemical composition was obtained:

$$
\begin{gathered}
K=82.0295+0.7727 X_{1}^{3}+6.7041 X_{1}^{2}+9.3974 X_{1}+1.8638 X_{2}^{2}+12.3974 X_{2}+ \\
+7.4978 X_{3}^{2}+3.4375 X_{3}+75.8217 X_{4}^{2}+171.0485 X_{4} .
\end{gathered}
$$




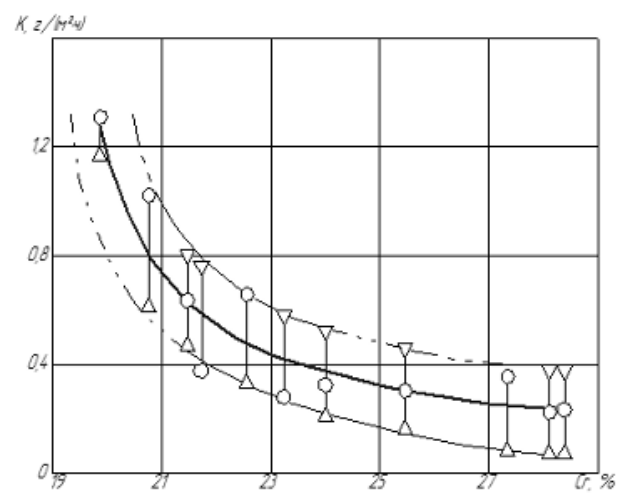

a)

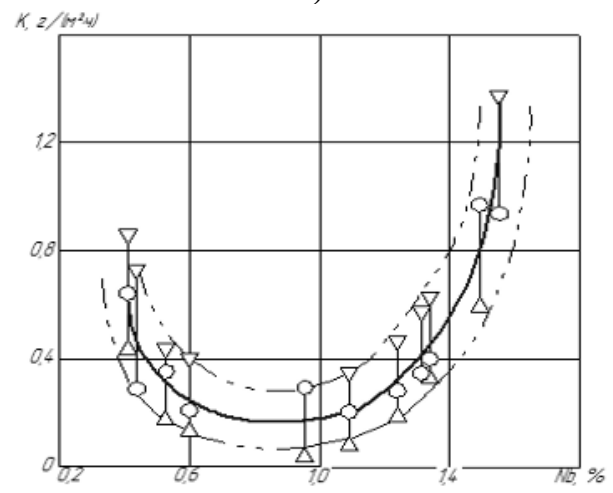

c)

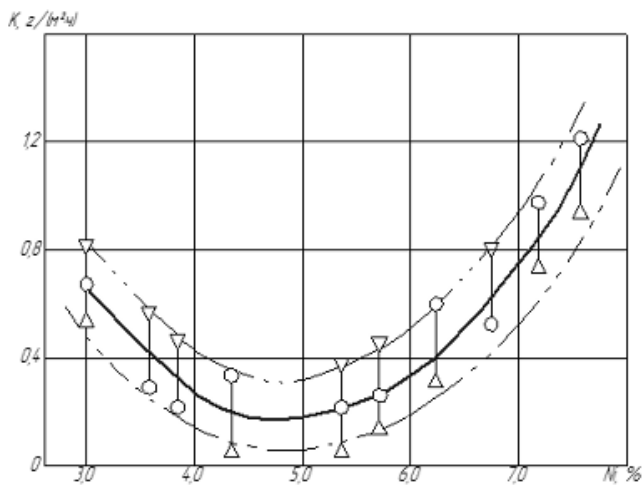

b)

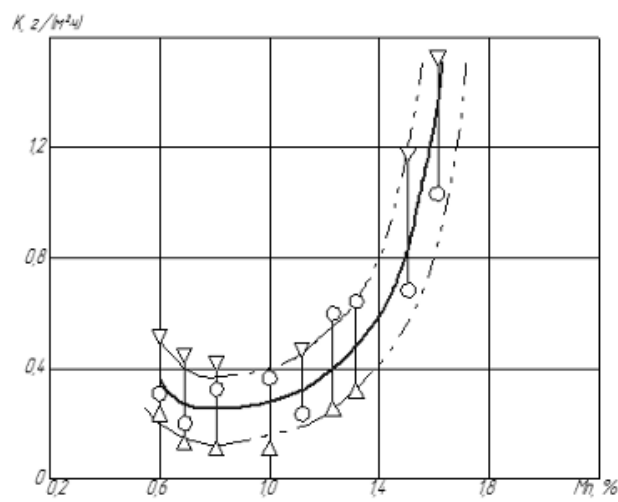

d)

Fig. 1. Dependence of the corrosion rate of the deposited metal on the content of chromium (a), nickel (b), niobium (c), manganese (d)

Further processing of the experimental results consisted in finding the optimal chemical composition of the deposited metal with high corrosion resistance in the urea medium by the steepest ascent method (the Box-Wilson method). According to the flowchart (Fig. 2), a computer program was compiled in FORTRAN 2018. The program included the calculation of the corrosion rate using the regression equation for the deposited metal, the chemical composition of which was selected by sequentially increasing the number of variable elements with a given step from the minimum to the maximum value. In addition, the corrosion rate was calculated for various values of the varied parameters.

The data block of the corrosion rate of the deposited metal, calculated depending on the number of elements, consisted of 1,260 variants. The best corrosion resistance was exhibited by the deposited metal containing 28.0 pts. wt. of $\mathrm{Cr}, 8.0$ pts. wt. of Ni, 0.5 pts. wt. of $\mathrm{Nb}, 1.1$ pts. wt. of $\mathrm{Mn}$.

It is known that at $\geq 25.0$ pts. wt. of chromium, the plastic properties of the deposited metal become deteriorated. Corrosion resistance in urea melt increases insignificantly when the amount of chromium changes in the range of 25.0-29.0 pts. wt. (Fig. 1, a). Therefore, its content was limited to the items from 26.0 to 28.0 pts. wt. To obtain the optimal phase composition, it is necessary to ensure the nickel content in the range of 7.0-8.0 pts. wt. 


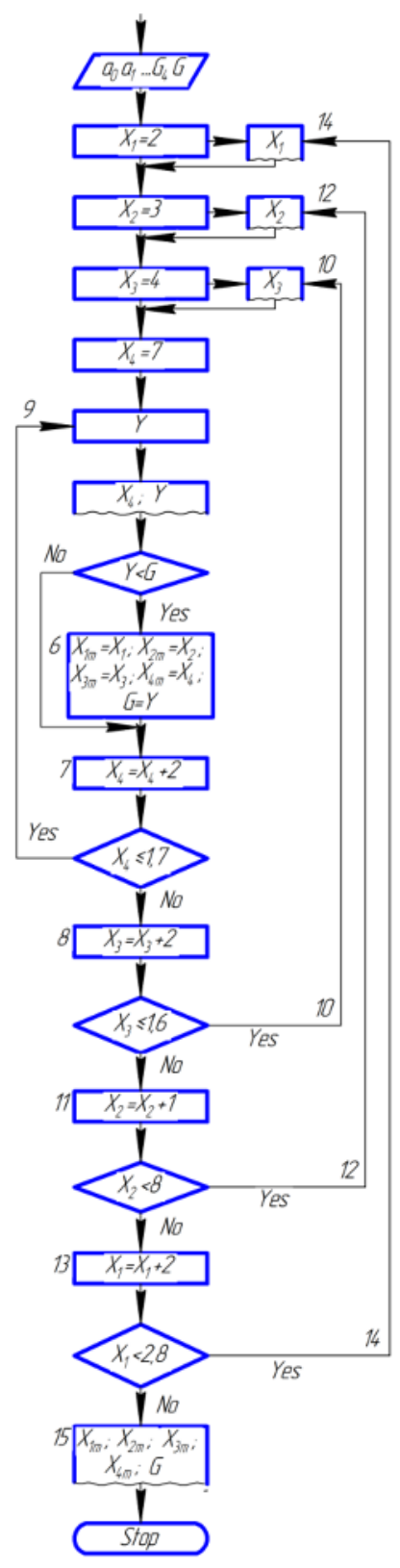

Fig. 2. Flowchart of the search program for the optimal chemical composition of the deposited metal

The results of corrosion tests of deposited samples (27.0 pts. wt. of Cr, 7.0 pts. wt. of $\mathrm{Ni}, 0.5$ pts. wt. of $\mathrm{Nb}, 1.1$ pts. wt. of $\mathrm{Mn}$ ) showed that the corrosion rate made 0.1915 $\mathrm{g} /\left(\mathrm{m}^{2} \cdot \mathrm{h}\right)$ and was close to the corrosion rate of the deposited metal of the optimal composition calculated by the regression equation $\left(0.1902 \mathrm{~g} /\left(\mathrm{m}^{2} \cdot \mathrm{h}\right)\right)$. The deposited samples of developed cast rods, after tests in the urea melt, showed no traces of local corrosion, which was general in its nature. 
Based on the calculated and experimental data, the following optimal chemical composition of the deposited metal (pts. wt.) was determined: carbon 0.02-0.06; silicon 0.20.8 ; manganese $0.5-1.2$; chromium 25.0-29.0; nickel 5.0-8.0; molybdenum 2.5-3.5; nitrogen $0.05-0.3$; cobalt $0.15-0.4$; niobium $0.45-0.75$; cerium $0.01-0.1$; copper $1.25-2.5$; while iron made the rest.

To determine the process through, comparative tests of coatings deposited with experimental cast rods and UONI-13/N1-BK electrodes were carried out at a deformation rate of $0-30 \mathrm{~mm} / \mathrm{min}$ in the shortness temperature range of $1,000-1,450^{\circ} \mathrm{C}$. The strength of the metal deposited with experimental cast rods is high. The critical deformation rate of the deposited metal by the developed cast rods in the brittleness temperature range made 26 $\mathrm{mm} / \mathrm{min}$, while using UONI-13/N1-BK electrodes $-14 \mathrm{~mm} / \mathrm{min}$.

\section{Conclusions}

1. A mathematical model of the dependence of the corrosion rate of the deposited metal on the content of alloying elements (chromium, nickel, niobium, manganese) is proposed.

2. High corrosion resistance in urea medium, workability, strength and ductility are possessed by the deposited metal containing (pts. wt.) carbon 0.02-0.06; silicon 0.2-0.8; manganese 0.5-1.2; chromium 25.0-29.0; nickel 5.0-8.0; molybdenum 2.5-3.5; nitrogen $0.05-0.3$; cobalt $0.15-0.4$; niobium $0.45-0.75$; cerium $0.01-0.1$; copper $1.25-2.5$; while iron makes the rest.

The research was carried out with the financial support of the Kuban Science Foundation in the framework of the Scientific Project No. МФИ-20.1/262.

\section{References}

1. Corrosion Resistance of Welded Joints of Economically Alloyed Stainless Steels in Corrosive Medium. B.V. Koshkin, Z.A. Sidlin, A.V. Mosolov, O.I. Steklov. Corrosion Protection in Chemical Industry: Information Overview. M.: Scientific Research Institute of Technical and Economic Research in Chemical Complex, 1981. 40 p.

2. Influence of Heat Input on Corrosion Resistance of Welded Joints of Austenitic-Ferritic Steel of 10Kh21N5T Type. Yu.N. Savonov, A.G. Alexandrov, V.M. Ruban, V.P. Loktionov. Welding Production. 1987. No. 7. P. 9-11.

3. Composition for Surfacing Parts. Nazarko, A.S., Plomodyalo, R.L., Demonov, M.S. Patent for Invention RU 2705273 C1, 07.11.2019. Application No. 2018147607 dated 28.12.2018.

4. Influence of Heat Treatment on Structure and Properties of Coatings Obtained by Surfacing with Cast Rods. Plomodyalo, R.L., Nazarko, A.S., Shtokolov, S.S. Welding and Diagnostics. 2021. No. 1. P. 37-40.

5. Protodyakonov, M.M., Teder, R.I. Methodology for Rational Planning of Experiments. M.: Nauka, 1970. 296 p.

6. Draper, N., Smith, G. Applied Regression Analysis. M.: Statistika, 1973. 392 p. 